\title{
OVÁRIOS POLICÍSTICOS EM MENINAS E ADOLESCENTES NORMAIS: UMA AVALIAÇÃO ULTRA-SONOGRÁFICA*
}

\author{
Rosimere J.Teixeira', Valéria C.G. Silva ${ }^{2}$, Josele R. Freitas ${ }^{3}$, Jodélia L.M. Henriques ${ }^{4}$, \\ Marília M. Guimarães ${ }^{5}$
}

Resumo Realizamos ultra-sonografia pélvica em tempo real e de alta resolução em 140 meninas com idade entre dois e 18 anos, para descrever a prevalência de ovários policísticos durante o desenvolvimento puberal normal. O volume dos ovários foi calculado e sua estrutura classificada como homogênea, microcística, multicística, policística e folicular. $\mathbf{O}$ volume aumentou e a frequiência das classes ovarianas variou de acordo com o status puberal. Os ovários eram de aspecto policístico em $8 \%$ (duas meninas pré-puberais e nove pós-puberais), com volume normal em 8/11 pacientes. Consideramos que a utilização do volume como critério diagnóstico de ovários policísticos pode ser de difícil interpretação durante este período e enfatizamos a importância da avaliação da ecogenicidade do estroma pelo ultra-som. Uma hipótese atrativa, mas que necessita de confirmação através de estudos longitudinais, é se essas meninas com ovários policísticos na ultra-sonografia serão destinadas, em alguns casos, a tornar-se adultas com a síndrome dos ovários policísticos.

Unitermos: Ovários policísticos. Ultra-sonografia. Meninas normais.

Abstract Polycystic ovaries in normal children and adolescent girls: ultrasound evaluation.

Real-time high-resolution ultrasonography of the pelvic organs was performed in 140 healthy females aged 2-18 years. The aim of this study was to establish the prevalence of polycystic ovaries during normal pubertal development. The volume of the ovaries was calculated and ovarian structure was classified as homogeneous, microcystic, multycystic, polycystic and follicular. The volume of the ovaries increased and the ovarian structure varied according to sexual development. The ovaries exhibited a polycystic appearance in $8 \%$ of the cases (two prepubertal and nine postpubertal girls). The volume of the ovaries was normal in 8/11 patients with polycystic ovaries. We consider that the using of the volume of the ovaries as a diagnostic criterion for polycystic ovaries is difficult to interpret and we emphasize the importance of the evaluation of the ovarian stroma with ultrasound. An attractive hypothesis that needs confirmation by longitudinal studies is wether girls with ultrasound diagnosed polycystic ovaries are at risk of developing polycystic ovarian syndrome in adulthood.

Key words: Polycystic ovaries. Ultrasound. Healthy girls.

\section{INTRODUÇÃO}

A síndrome dos ovários policísticos (SOP) é a causa mais comum de hiperandrogenismo na mulher adulta e, em sua forma clássica, é caracterizada por irregularidade menstrual, hirsutismo, obesidade, níveis plasmáticos elevados dos androgênios e do hormônio luteotrófico e ovários policísticos (OP) ao ultra-som ${ }^{(\mathbf{1})}$.

\footnotetext{
* Trabalho realizado no Hospital Universitário Pedro Ernesto da Universidade do Estado do Rio de Janeiro (HUPE-UERJ) e no Hospital Universitário Clementino Fraga Filho da Universidade Federal do Rio de Janeiro (HUCFFUFRJ), Rio de Janeiro, RJ.

1. Doutora em Endocrinologia e Professora Visitante do HUPE-UERJ.

2. Professora Assistente de Radiologia do HUPE-UERJ. 3. Professora Assistente de Ginecologia do HUPE-UERJ.

4. Professora Adjunta de Endocrinologia do HUPE-UERJ. 5. Professora Adjunta de Endocrinologia do HUCFFUFRJ.

Endereço para correspondência: Dra. Rosimere J.Teixeira Rua Silva Rabelo, 10, sala 307, Meier. Rio de Janeiro, RJ, 20735080. E-mail: rosijt@fst.com.br

Aceito para publicação em 16/5/2001.
}

Nos últimos anos vem aumentando muito o interesse na SOP, porque essas mulheres podem apresentar infertilidade e importante morbidade, principalmente por estar associado com um maior risco de desenvolver doença cardiovascular e diabetes mellitus ${ }^{(\mathbf{1 , 2})}$

A SOP pode ser bem mais comum na adolescência do que se imagina. As alterações hormonais e/ou mudanças morfológicas ovarianas parecem começar ainda na infância ${ }^{(3-5)}$, e o aumento dos níveis do fator de crescimento semelhante à insulina I (IGF-I) e da insulina durante a puberdade pode estar relacionado com o aumento da prevalência da SOP nesta fase $^{(6-8)}$. Entretanto, os relatos da literatura sobre o desenvolvimento normal dos ovários na infância, por meio da ultra-sonografia (US), são limitados e não costumam descrever em detalhes as alterações morfológicas que ocorrem durante a puberdade ${ }^{(9-11)}$. Além disso, na prática diá- ria não existem critérios bem estabelecidos para descrever a estrutura ovariana. Os termos "ovários microcísticos, micropolicísticos e policísticos" são comumente usados, mas de maneira confusa, sempre que se encontram cistos periféricos ao ultra-som.

Este estudo tem como objetivo descrever a prevalência de OPs durante o desenvolvimento puberal normal.

\section{CASUÍSTICA E MÉTOdos}

Trata-se de um estudo descritivo e de corte transversal, que foi aprovado pelo Comitê de Ética do Hospital Universitário Pedro Ernesto da Universidade Estadual do Rio de Janeiro. Foram selecionadas 140 meninas pré e pós-puberais saudáveis, entre dois e 18 anos incompletos, encaminhadas ao ambulatório de endocrinologia pelo serviço de ginecologia infanto-puberal para participarem deste 
estudo. Foram excluídos os casos com desordens puberais ou endócrinas, irregularidade menstrual - definida como ciclo < 21 ou > 40 dias -, doenças crônicas, uso de pílula anticoncepcional ou outras drogas. Foram avaliados os estádios puberais de Tanner, a idade da menarca e o ciclo menstrual, a estatura, o peso e o índice de massa corporal ( $\mathrm{IMC}=\mathrm{kg} / \mathrm{m}^{2}$ ). Foi considerado como sinal clínico de puberdade a presença de mamas - estádio de Tanner II em diante.

A US pélvica foi realizada e interpretada sempre por um dos autores, através do método convencional com bexiga repleta, obtida após a administração oral de fluidos e retenção urinária voluntária. Foram utilizados os seguintes equipamentos ultra-sonográficos de alta resolução em tempo real: Logic $500 \mathrm{GE}$ com sonda de 3,5 MHz e Hitachi modelo EUB 305 com transdutor de 3,5 ou 5,0 $\mathrm{MHz}$. O volume ovariano (VO) foi calculado por meio da fórmula do elipsóide:

$$
\mathrm{V}=\mathrm{L} \times \mathrm{AP} \times \mathrm{T} \times 0,5233
$$

onde: $\mathrm{V}=$ volume; $\mathrm{L}=$ diâmetro longitudinal; AP = diâmetro ântero-posterior e T $=$ diâmetro transversal.

A estrutura ovariana foi dividida em cinco classes, como se vê no Quadro 1. A classificação aqui proposta é baseada no número de cistos, volume dos folículos e estroma ovariano, sendo uma modificação de outras já relatadas previamente ${ }^{(9-11)}$. A medida e a classe de cada ovário foram consideradas separadamente, devido às variações existentes entre volume, número e diâmetro dos folículos por par de ovário. Os ovários com macrocistos (cistos > $20 \mathrm{~mm}$ ) foram excluídos para a avaliação da média do VO.

Para a análise estatística foram utilizados os testes paramétrico Anova e nãoparamétrico qui-quadrado $\left(\chi^{2}\right)$ com correção de Yates, quando indicado. Os resultados foram expressos em média \pm desvio padrão (SD), adotando-se o nível de significância de $95 \%$, onde $\mathrm{p}<0,05$.

\section{RESULTADOS}

A US pélvica foi realizada em 140 meninas, sendo 57 em estádio de Tanner I $(6,95 \pm 1,97$ anos; IMC $=16,98 \pm 2,64)$,
Quadro 1 Classificação da estrutura ovariana.

\begin{tabular}{|c|cc|}
\hline Classes & Descrição da estrutura ovariana \\
\hline 1 & $\Rightarrow$ Homogênea - Aspecto sólido ou presença de até três cistos com diâmetro \\
menor que $9 \mathrm{~mm}$.
\end{tabular}

Classificação modificada e baseada nos dados de Adams et al!(20), Salardi et al!(14) e Nobels e Dewailly(7).
35 em estádio de Tanner II-III $(10,41 \pm$ $1,28$ anos; IMC $=18,15 \pm 3,10)$ e 48 em estádio de Tanner IV-V $(13,10 \pm 2,01$ anos; IMC $=20,93 \pm 5,24)$. No grupo puberal a menarca ocorreu em 31 meninas (11,8 $\pm 1,4$ anos), sendo a US realizada no primeiro ano da menarca em $12 \mathrm{e}$ após um a cinco anos em 19 casos.

Foi observado aumento significativo do VO com o avanço dos estádios puberais (Tanner $\mathrm{I}=0,9 \pm 0,5 \mathrm{~cm}^{3}$, Tanner IIIII $=2,2 \pm 1,3 \mathrm{~cm}^{3}$ e Tanner IV-V $=4,8 \pm$ $2,2 \mathrm{~cm}^{3} ; \mathrm{p}<0,001$ ) (Figura 1). A distribuição das classes ovarianas variou de acordo com o estádio puberal ( $\mathrm{p}<0,005)$ (Figura 2). Em 95\% dos casos a classe ovariana foi a mesma por par de ovários. Em 11 casos um dos ovários não foi visibilizado (4\%), mais comumente o esquerdo $(9 / 11)$.

A estrutura policística (classe 4) (Figura 3) foi encontrada em 11 pacientes, sendo dois casos (4\%) no estádio prépuberal e nove casos (11\%) no estádio pós-puberal, mas somente após os oito anos de idade. As principais características clínicas dos casos com OPs são mostradas na Tabela 1. O VO foi aumentado (> $2 \mathrm{SD}$ ) para o estádio puberal em apenas três casos (Figura 4).

\section{DISCUSSÃO}

As mudanças morfológicas nos ovários das pacientes com SOP podem ser consideradas como um exagero daquelas que ocorrem na puberdade normal ${ }^{(7)}$. Em estudo prévio descrevemos que a estrutura ovariana depende da maturidade biológica, isto é, varia de acordo com a idade e o estádio puberal. O ovário normal passa por várias fases de desenvolvimento e assume, transitoriamente, os aspectos homogêneo, microcístico, multicístico e folicular, sugerindo que o número de cistos aumenta à medida que ocorre o avanço puberal. O ovário homogêneo é típico do estádio pré-puberal, sendo de aspecto sólido em apenas 11\% dos casos. Com o início da puberdade, os ovários homogêneos não são mais visibilizados e ocorre uma mudança na estrutura, com predomínio dos microcistos e multicistos. Os folículos dominantes ou préovulatórios são observados principalmente próximo à menarca, quando a maturação sexual é mais avançada ${ }^{(12)}$. Os multicistos parecem ocorrer como uma resposta normal da maturação ovariana, antes da seleção do folículo dominante, e o diâmetro máximo dos folículos au-
Tabela 1 Características clínicas dos casos com ovários policísticos.

\begin{tabular}{|l|c|c|c|c|c|c|c|c|c|c|c|}
\hline & \multicolumn{10}{|c|}{ Casos } \\
\cline { 2 - 11 } & 1 & 2 & 3 & 4 & 5 & 6 & 7 & 8 & 9 & 10 & 11 \\
\hline IC (anos) & 8,5 & 9,3 & 11,4 & 11,8 & 11,8 & 10,7 & 11,5 & 11,7 & 9,4 & 12,0 & 16,0 \\
IMC (kg/m²) & 14,7 & 16,5 & 16,9 & 14,2 & 16,3 & 15,0 & 15,6 & 13,9 & 16,7 & 15,3 & $32,6^{*}$ \\
$\begin{array}{l}\text { Estádio } \\
\text { de Tanner } \\
\begin{array}{l}\text { Menarca } \\
\text { (idade - anos) }\end{array}\end{array}$ & I & I & II & II & II & III & IV & IV & IV & V & V \\
\hline
\end{tabular}

IC, idade cronológica; IMC, índice de massa corporal (*IMC > percentil 95 do gráfico de Rolland-Cachera ${ }^{(25)}$ ). 


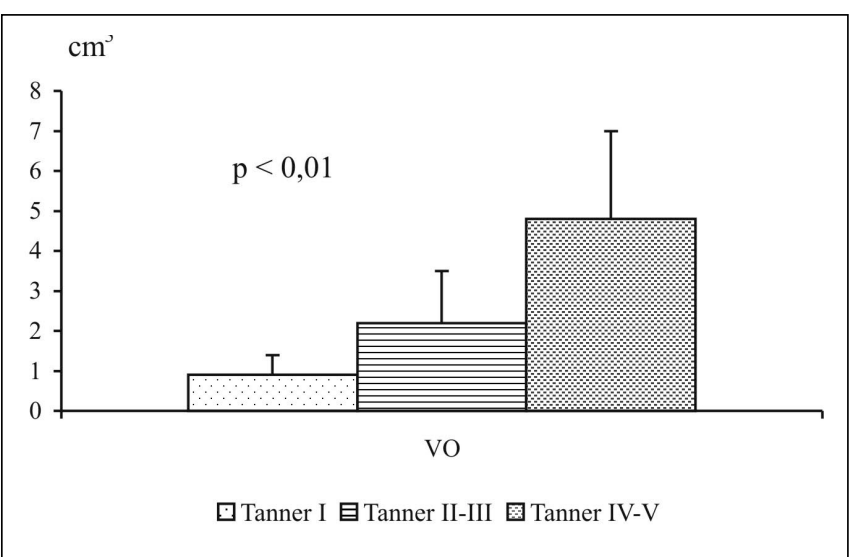

Figura 1. Volume ovariano (VO) por estádio de desenvolvimento puberal.

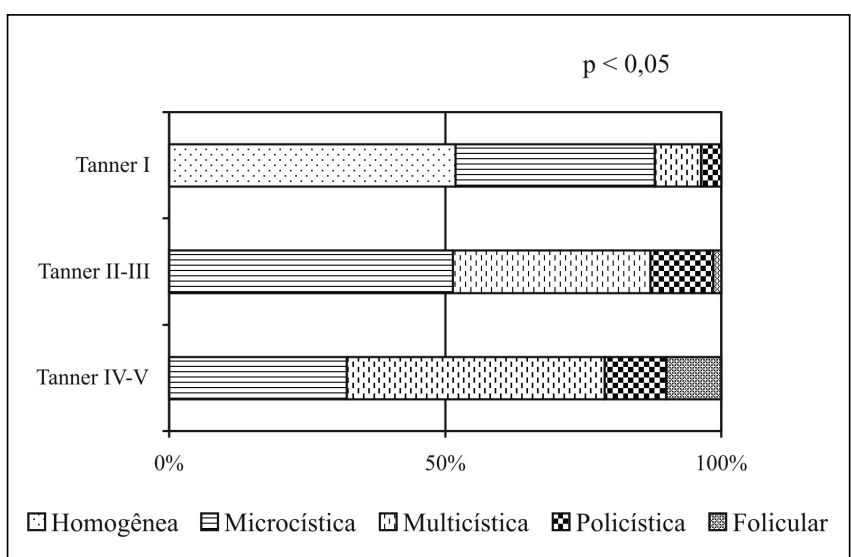

Figura 2. Porcentual das classes ovarianas de acordo com o estádio de desenvolvimento puberal.

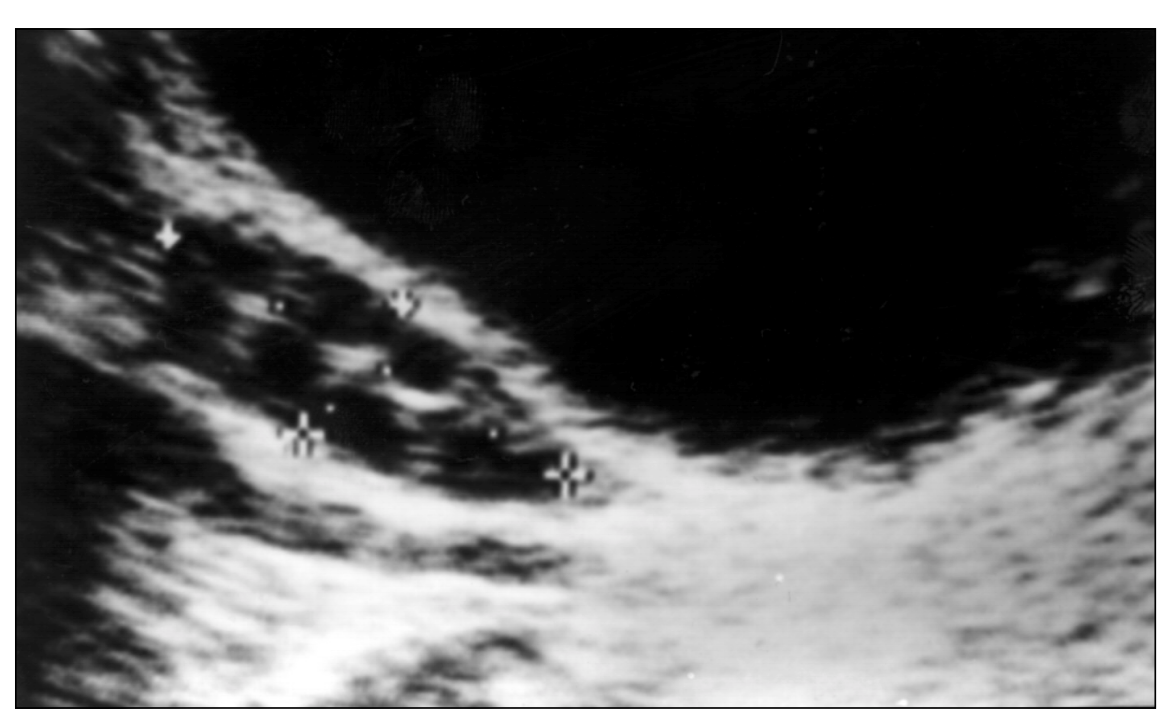

Figura 3. Estrutura ovariana policística: mais de dez cistos menores que $9 \mathrm{~mm}$ e estroma ovariano hiperecogênico.

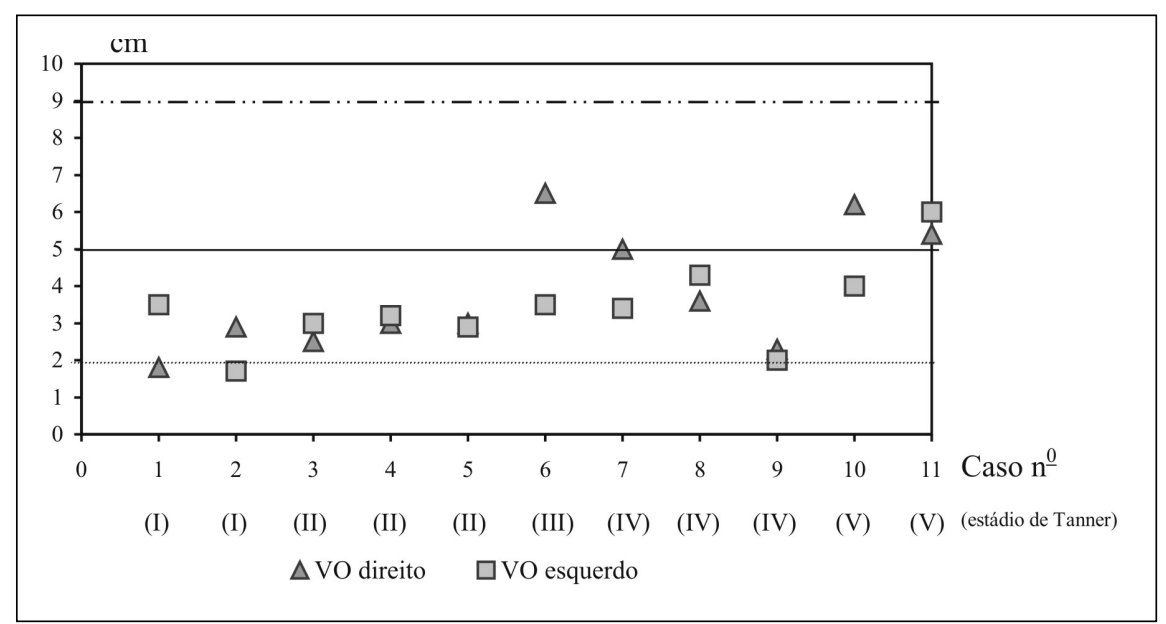

Figura 4. Volume ovariano dos casos com estrutura policística. As linhas .........., _ _ e _.....representam 2 SD do normal para os estádios puberais, isto é, $2,0 \mathrm{~cm}^{3}$ para 0 estádio de Tanner I, $5,0 \mathrm{~cm}^{3}$ para 0 estádio de Tanner II-III e $9,0 \mathrm{~cm}^{3}$ para 0 estádio de Tanner IV-V, respectivamente. Apenas os casos 1, 2 e 6 apresentaram pelo menos um ovário com volumes aumentados (> 2 SD) para o estádio puberal. menta com a progressão da puberdade ${ }^{(3,}$ ${ }^{11-15)}$. Assim que o padrão adulto de secreção hormonal é atingido, a resposta ovariana às gonadotrofinas torna-se bastante reduzida e, então, os multicistos desaparecem $^{(15,16)}$. Possivelmente, neste momento, os ovários reassumem o aspecto normal, isto é, a estrutura homogênea ${ }^{(17)}$.

Como descrito, o ovário passa por várias fases de desenvolvimento, que são dependentes do estímulo das gonadotrofinas. Mesmo assim, os OPs estão presentes em poucos casos na infância, quando a concentração de gonadotrofinas é baixa. Bridges et al. encontraram prevalência de OPs que aumentou de $6 \%$ para $26 \%$ dos seis aos $15 \operatorname{anos}^{(3)}$, enquanto nossos achados são de $4 \%$ nas meninas pré-puberais e de $11 \%$ nas pós-puberais.

Nem sempre os critérios ultra-sonográficos utilizados para o diagnóstico da SOP são os mesmos. Stein e Leventhal descreveram a SOP, enfatizando a presença de ovários grandes bilateralmente, com múltiplos cistos de 3-7 mm em mulheres com amenorréia secundária, hirsutismo e obesidade ${ }^{(18)}$. Em nossa casuística, a obesidade foi observada em apenas um dos 11 casos. Devemos lembrar que crianças e adolescentes obesos têm maior propensão de ser obesos quando adultos e que o excesso de peso na infância está associado à $\mathrm{SOP}^{(\mathbf{1 9})}$.

Outra definição ultra-sonográfica amplamente utilizada e a escolhida em nosso estudo é a descrita por Adams et al., que definem como OP a presença de dez ou mais cistos de 2-8 mm de diâmetro envoltos por aumento do estroma ${ }^{(20)}$. Vem 
sendo também utilizada a classificação de Nobels et al. para ovários multicísticos quando, apesar da presença de dez ou mais cistos, o estroma não está aumentado $^{(7)}$. Parece que a diferenciação entre ovários multicísticos e policísticos tem alguma importância clínica, como demonstrado por Hohleuwerger et al., que consideram a presença dessas estruturas como dois extremos das alterações anatômicas e de um amplo espectro clínico e hormonal presentes na $\mathrm{SOP}^{(21)}$. Entretanto, em estudo posterior e ainda em vias de publicação, esses autores encontraram a estrutura multicística em $47 \%$ das mulheres normais, indicando que o número de cistos é um parâmetro de baixa especificidade para o diagnóstico da SOP. Este fato também é reforçado por outros estudos, que demonstram que os ovários multicísticos são comuns tanto em adolescentes normais ${ }^{(\mathbf{1 2})}$ como naquelas com irregularidade menstrual ${ }^{(22)}$.

Alguns autores consideram a análise do VO maior que 9-10 $\mathrm{cm}^{3}$ importante para o diagnóstico de SOP, tanto em adolescentes quanto na mulher adulta ${ }^{(\mathbf{1}, 6,23)}$. Segundo Avvad, a estrutura policística e o aumento do VO são achados ultra-sonográficos comuns em adolescentes com irregularidade menstrual nos três primeiros anos, naquelas com irregularidade menstrual persistente e nas com $\mathrm{SOP}^{(22)}$.

No presente estudo, a maioria das pacientes com OPs ainda não havia menstruado e as duas pacientes com história de menarca (casos 10 e 11) apresentavam ciclos regulares e volumes normais $(<9$ $\mathrm{cm}^{3}$ ), ao passo que somente três casos apresentaram pelo menos um ovário com volume aumentado para o estádio puberal. Alguns autores consideram que o VO é relacionado com o grau da hiperplasia do estroma ${ }^{(1,24)}$, e como o estroma é o principal local da produção dos androgênios, Hohleuwerger et al. acreditam que o aumento da ecogenicidade do estroma é o parâmetro ultra-sonográfico mais específico para a classificação de $\mathrm{OP}^{(21)}$. $\mathrm{Na}$ infância, o VO apresenta um crescimento contínuo $^{(\mathbf{1 0 - 1 3 )}}$, por isso consideramos que a utilização do volume como critério para a definição de OP pode ser de difícil interpretação durante este período e preferimos definir em relação à ecogenicidade do estroma.

Em resumo, OPs podem ser encontrados em meninas e adolescentes com desenvolvimento puberal normal, embora não seja achado ultra-sonográfico muito comum. Na infância o ovário apresenta crescimento contínuo e progressivo, logo a utilização do volume como critério diagnóstico pode ser de difícil interpretação e a avaliação da ecogenicidade do estroma torna-se o principal parâmetro para a classificação ultra-sonográfica de OPs. Como a SOP geralmente se desenvolve na adolescência e ainda não é claro se a aparência policística ao ultra-som reverte ao normal, então essas meninas devem ser acompanhadas para se afastar uma provável evolução para a SOP.

\section{REFERÊNCIAS}

1. Lobo RA. Polycystic ovary syndrome/ hyperandrogenic chronic anovulation. Adv Endocrinol Metab 1995;6:167-91.

2. Mantzoros CS, Flier JS. Insulin resistance: the clinical spectrum. Adv Endocrinol Metab 1995; 6:193-232.

3. Bridges NA, Cooke A, Healy MJR, Hindmarsh PC, Brook CGD. Standards for ovarian volume in childhood and puberty. Fertil Steril 1993;60: 456-60.

4. Ehrmann DA, Barnes RB, Rosenfield RL. Polycystic ovary syndrome as a form of functional ovarian hyperandrogenism due to dysregulation of androgen secretion. Endocrine Rev 1995;16: 322-53.

5. Hohleuwerger R, Teixeira RJ, Bordallo MAN, Gazolla HM. Perfil androgênico e resistência à insulina: adolescentes com síndrome de ovários policísticos. Gynaecia 1997;3:4-8.

6. Giorlandino C, Gleicher N, Taramanni C, et al. Ovarian development of the female child and adolescent: I. Morphology. Int J Gynaecol Obstet 1989;29:57-63.

7. Nobels F, Dewailly D. Puberty and polycystic ovarian syndrome: the insulin/insulin-like growth factor I hypothesis. Fertil Steril 1992;58:655-66.

8. Yen SSC, Laughlin GA, Morales AJ. Interface between extra- and intraovarian factors in polycystic ovarian syndrome. Ann NY Acad Sci 1993;687: 98-111.

9. Balen A, Dunger D. Pubertal maturation of the internal genitalia. Ultrasound Obstet Gynecol $1995 ; 6: 164-5$
10. Ivarsson SA, Nilsson KO, Persson PH. Ultrasonography of the pelvic organs in prepubertal and postpubertal girls. Arch Dis Child 1983;58:352-4.

11. Orsini LF, Salardi S, Pilu G, Bovicelli L, Cacciari E. Pelvic organs in premenarcheal girls: real-time ultrasonography. Radiology 1984;153:113-6.

12. Teixeira RJ, Silva VCG, Freitas JR, Henriques JLM, Guimarães MM. Ultra-sonografia pélvica em 140 meninas normais pré e pós-puberais. Arq Bras Endocrinol Metab 1999;43:210-16.

13. Holm K, Laursen EM, Brocks V, Müller J. Pubertal maturation of the internal genitalia: an ultrasound evaluation of 166 healthy girls. Ultrasound Obstet Gynecol 1995;6:175-81.

14. Salardi S, Orsini LF, Cacciari E, et al. Pelvic ultrasonography in girls with precocious puberty, congenital adrenal hyperplasia, obesity, or hirsutism. J Pediatr 1988;112:880-7.

15. Stanhope R, Adams J, Jacobs HS, Brook CGD Ovarian ultrasound assessment in normal children, idiopathic precocious puberty, and during low dose pulsatile gonadotrophin releasing hormone treatment of hypogonadotrophic hypogonadism. Arch Dis Child 1985;60:116-9.

16. Venturoli S, Porcu E, Fabbri R, Paradisi R, Orsini LF, Flamigni C. Ovaries and menstrual cycles in adolescence. Gynecol Obstet Invest 1984;17:219_ 22.

17. Venturoli S, Porcu F, Fabbri R, et al. Ovarian multifollicularity, high $\mathrm{LH}$ and androgen plasma levels, and anovulation are frequent and strongly linked in adolescent irregular cycles. Acta Endocrinol 1986;111:368-72.

18. Stein JF, Leventhal ML, Ill C. Amenorhea associated with bilateral polycystic ovaries. Am J Obstet Gynecol 1935;29:81-9.

19. Schonfeld-Warden N, Warden CH. Obesidade pediátrica: uma visão global da etiologia e do tratamento. Clín Pediátr Am Norte 1997;2:343-66.

20. Adams J, Polson DW, Franks S. Prevalence of polycystic ovaries in women with anovulation and idiopathic hirsutism. Br Med J 1986;293:355-9.

21. Hohleuwerger R, Silva VCG, Bordallo MAN, Dimetz T, Gazolla HM. Espectro ultra-sonográfico no hiperandrogenismo: ovários policísticos $\times$ ovários multicísticos. Rev Bras Ginecol Obstet 1996;18:637-41.

22. Avvad CK. Irregularidade menstrual na adolescência - manifestação clínica precoce da síndrome dos ovários policísticos? [Tese de Mestrado]. Rio de Janeiro: Universidade do Estado do Rio de Janeiro, 2000.

23. Herter LD, Magalhães JA, Spritzer PM. Association of ovarian volume and serum LH levels in adolescent patients with menstrual disorders and/or hirsutism. Braz J Med Biol Res 1993;26:1041-6.

24. Shoham Z, Conway GS, Patel A, Jacobs HS. Polycystic ovaries in patients with hypogonadotrophic hypogonadism: similarity of ovarian response to gonadotropin stimulation in patients with polycystic ovarian syndrome. Fertil Steril 1992; 58:37-45.

25. Rolland-Cachera MF. Onset of obesity assessed from the weight/stature ${ }^{2}$ curve in children: the need for a clear definition (letter comment). Int J Obes Relat Metab Disord 1993;17:245-6. 